Article

\title{
Innovative Reconfigurable Metasurface 2-D Beam-Steerable Reflector for 5G Wireless Communication
}

\author{
David Rotshild *, Efraim Rahamim and Amir Abramovich (D) \\ Department of Electrical and Electronic Engineering, Ariel University, Ariel 40700, Israel; \\ efraim.rahamim@msmail.ariel.ac.il (E.R.); amir007@ariel.ac.il (A.A.) \\ * Correspondence: david.rotshild@msmail.ariel.ac.il
}

Received: 23 June 2020; Accepted: 21 July 2020; Published: 24 July 2020

check for updates

\begin{abstract}
A tunable reflector component based on metasurface (MS) with a low profile and reduced mass is offered for indoor and outdoor 5G communication methods to overcome obstacles such as buildings, walls, and turns, and to allow wireless quasi-line of sight path communication at $37 \mathrm{GHz}$. Integrating varactors with MS unit cells allows tunability and reconfigurability. This approach was presented in many studies, with frequencies of up to $\mathrm{K}$-band. However, today, higher frequencies are used, especially in communication. This work presents the design of a reconfigurable MS reflector, at Ka-band frequencies, based on a new type of resonant unit cell, with uniformed reflection for wide-incident-angular-range, and a simple stimulating DC bias for each MS unit cell, which allows a two-dimensional (2-D) continuous reflection phase manipulation. The unit cell provides a dynamic reflection phase range of over $300^{\circ}$ at a wide bandwidth. Simulations of one-dimensional (1-D) and (2-D) at $37 \mathrm{GHz}$ are presented. A steering range of up to $\pm 48^{\circ}$ was obtained for azimuth or elevation. A simultaneous independent 2-D beam steering range of up to $\pm 10^{\circ}$ in azimuth and up to $\pm 5^{\circ}$ in elevation, allowing obstacles to overcome covering at a practical angular spatial cone of $20^{\circ}$ and $10^{\circ}$, is presented.
\end{abstract}

Keywords: beam steering; Ka-band; metasurface; tunable circuits and devices

\section{Introduction}

The demand to increase data communication rates leads to an increase in used frequencies, as seen in the development of the fifth generation (5G) of cellular communications, which uses millimeter waves (MMW) (30-300 GHz) for indoor, short-range links, and outdoor point to point links. The nature of wave propagation in MMW radiation is approximately like the ray-tracing model. It is affected by atmospheric conditions, specular reflections and multi-path, and directivity of transmitters and receivers [1]. Implementing tuning reflectors for the $5 \mathrm{G}$ wireless communication allows a quasi-line of sight paths and improves the link. In outdoor communication, it requires bypassing obstacles such as buildings and other constructions in urban areas or mountains, and more, in non-urban areas [2-4]. In indoor communication, it requires tunable reflectors to bypass walls and turns [5].

In addition, the implementation of $5 \mathrm{G}$ cellular communication requires tracking the user's location continuously and directing the MMW beam correctly. The tracking procedure is carried out nowadays using the existed $4 \mathrm{G}$ network. Knowing the exact user's location enables the base station to find the best trajectory using tunable reflectors, between the base station and the user [6].

However, low-cost, low profiles, reduced masses, remote-controlled, and efficient solutions are required due to the network density and the number of components. A metasurface (MS) solution, which can be programmed remotely by the base station to bring the beam optimally to the user with low weight, low complexity, and low cost, is offered in this work. 
Metasurfaces (MSs), and especially reconfigurable MSs, arouse great interest in science. There are many interdisciplinary applications where MSs play an important role [7]. For example, a millimeter-wave MS perfect absorber was designed to detect micro-poisons in drinking water [8], and a flat parabolic surface was used in W-band imaging and beam propagation [9]. These MSs are based on resonant unit cells and are limited to a narrow bandwidth around a fixed working frequency. Adding tunability to each unit cell enables a reconfigurable MS and expands the abilities and options of MS applications. Integrated electronic devices or tunable materials achieve the reconfigurability to the MS. For example, PIN switches, MEMS switches, liquid crystal or piezoelectric materials, and varactor diodes $[10,11]$. Using the switches, it becomes possible to connect and disconnect conductive elements on the unit cell, or between unit cells on the surface, thereby changing the electrical properties of the surface. Switches are conveniently integrated into standard PCB circuits. The PIN switch enables MS applications of up to $60 \mathrm{GHz}$ [12], with fast switching time, high reliability, low cost, and low control voltage switches $[10,13]$. Recently, the MEMS switch arouses attention due to its small size and linearity, but it has a relatively slow switching time, compared to the pin switch [14]. The discrete nature of switches prevents their use in applications where a continuous phase change is required. Liquid crystal changes the dielectric coefficient of a unit cell by tens of percent and can be realized in the surface without any external element in the front. This method is more difficult to implement and can be used at K-band and above [15]. However, it has a slow response time compared to switches and varactors [16]. The piezoelectric method allows limited flexibility proportional to the change in its physical size, which is also its obvious drawback [11]. The varactor diodes allow continuous and high tuning ratio capacitance, constant Gamma for linear tuning, a convenient PCB assembly, and very low consuming power [17].

Reconfigurable MSs were implemented for various applications, such as: reconfigurable reflect arrays [10,18,19], reconfigurable antennae [20], leaky-wave antennae [21], tunable filters and absorbers [22], parabolic mirrors with a controlled focus [23], and tuning polarization rotators [24]. Reconfigurable MSs based on varactor diodes were realized at X-band and below [21-28]. For Ku-band frequencies, only simulation works have been published [29,30]. Adjusting the MS component by developing appropriate unit cells for the new, used frequencies is required.

However, manufacturing MS at higher frequencies bands such as K-band and Ka-band requires a decrease in unit cell dimensions. Thus, the varactor size becomes a significant part of the front MS area causing more absorption and diffusion. Design and consideration of a new reconfigurable MS reflector at K-band are presented and simulated. This MS reflector allows a simultaneous independent 2-D continuous steering ability of the reflection radiation and is suitable for indoor and outdoor 5G communication methods, to overcome obstacles such as buildings, walls, and turns. Using the continuous reflection phase method compared to the discrete reflection phase method, when using switches, allows avoiding beam steering quantization loss, of up to $3 \mathrm{~dB}$ [18]. To the best of our knowledge, such a 2-D continuous tunable MS for K-band frequencies has neither been demonstrated nor published. The paper is organized as follows. Section 2 describes the general resonant MS unit cell principles and parameters based on effective impedance surface model. Section 3 introduces the steering MS refractor mathematical method, based on the phased array theory. Section 4 describes the unit cell and MS design principles and considerations. Section 5 presents simulations results and analyzes the unit cell reflection and the dynamic reflection phase range. Section 6 presents the reconfigurable MS reflector steering simulations results in 1-D and 2-D. Section 7 concludes this paper.

\section{Effective Impedance Surface Model of Unit Cells Array}

Reflective MSs are based on resonance unit cells, which are smaller than the working radiation wavelength. A basic equivalent unit cell circuit is a parallel resonance circuit. When the unit cells are arranged in a 2-D periodic form, the MSs are characterized by effective impedance surface [25] and are shown in Equation (1):

$$
Z_{s}=\frac{j \omega L}{1-\omega^{2} L C}
$$


where $L$ is the inductance and $C$ is the total capacitance of each unit cell. The values of $C$ and $L$ are determined by the unit cell geometry, materials, varactor parameters, and PCB properties. The parallel resonance frequency $f_{\text {res }}$ of the circuit model is shown in Equation (2)

$$
f_{\text {res }}=\frac{1}{2 \pi \sqrt{L C}}
$$

The bandwidth of the resonance frequency [31] is shown in Equation (3)

$$
B W=1 /\left(R C \omega_{0}\right)=\left(L \omega_{0}\right) / R \propto L / C
$$

where $R$ is the dissipation resistive part of the unit cell. This kind of surface is also known as High Impedance Surface (HIS) [32] or Perfect Magnetic Conductor (PMC) [33]. For a resonance frequency, this surface reflects incident radiation at $0^{\circ}$ phase in contrast to a normal metal surface, which reflects the radiation at $180^{\circ}$ phase. The reflection phase changes around $f_{\text {res. }}$. Far below the $f_{\text {res }}$, the surface reflects with a phase shift of $180^{\circ}$, like a metal surface, and approaches $-180^{\circ}$ for frequencies far above resonance, when for a wide $B W$, the change moderates and vice versa. Varactor diodes inserted into the unit cell provide variable capacitance $C_{d}$ to enable tunability [25-30]. The unit cell reflection phase value around the resonance frequency changes significantly. Thus, changing the $f_{\text {res }}$ around the working frequency changes the reflection phase.

Controlling the phase of each unit cell in the surface allows the design of spatial phase distribution, which leads to steer reflection radiation to the desired angle [25]. This approach is well known in the literature, as mentioned above, but MS-based varactor diodes are demonstrated only in Ku-band and below. However, MS realization at higher frequency bands such as K-band and Ka-band requires decreasing product $L C$ in Equation (2) to achieve higher $f_{\text {res. }}$. In the MS resonant unit cell, surface area size is proportional to intrinsic capacitance $C_{i n t}$, and thickness is proportional to intrinsic inductance $L_{\text {int }}$ [34]. Considering $C_{d}$ as a method of tuning, $C=C_{i n t}+C_{d}$, and $L=L_{i n t}$ in Equations (2) and (3). Reducing unit cell dimensions leads to production difficulty and challenge. The reduction in $L$ in the $L C$ product in Equation (2) is limited since it decreases bandwidth and increases the sensitivity to phase errors according to Equation (3). In addition, since the $Q$ factor is $Q=1 / \mathrm{BW}$ and total absorption is $R \times Q$ [34], decreasing $C$ in Equation (2) reduces $Q$ factor value, which reduces unit cell absorption. Thus, in decreasing product $L C$, the emphasis is $C$ reduction, which requires careful design since it is accompanied by reducing the unit cell area. Thus, the varactor size becomes a significant part of the front MS area, causing more absorption and diffusion. The proposed simple, inexpensive, and innovative unit cell has a low intrinsic capacitance $C_{i n t}$, which, along with using a small-size varactor with the lowest capacitance available on the market today [17], enables MS realization for Ka-band with reasonable dimensions, allowing conventional PCB manufacturing and varactor assembly.

\section{Mathematical Method of Steering MS Refractor}

According to the phased array theory, each unit cell location on the MS is defined at its center [35]. A 2-D surface on $\mathrm{XY}$ plane with spatial array arrangement of a fixed distance and a $90^{\circ}$ angle between the unit cells is defined as $\mathrm{S}\left(\mathrm{x}_{\mathrm{j}}, \mathrm{y}_{\mathrm{i}}\right), \mathrm{j}=1,2, \mathrm{M}$ and $\mathrm{i}=1,2, \mathrm{~N}$ when $\mathrm{N}$ and $\mathrm{M}$ are an integer leads to an array of $\mathrm{N} \times \mathrm{M}$ elements. The MS reflector side cross-section view scheme is described in Figure 1.

Figure 1 shows a reconfigurable MS reflector scheme. $\mathrm{L}_{1}, \mathrm{~L}_{2}$, and $\mathrm{L}_{\mathrm{N}}$ are incident rays towards the surface. Due to a planned gradual phase provided by the unit cells MS reflector, the rays are reflected at the desired angle $\theta$. The optical path difference (OPD) between the cells is defined as $\Delta L$ [9] and is shown in Equation (4)

$$
\Delta L=\Delta X \cdot \sin (\theta)
$$


where $\Delta X$ is the array constant. The conversion of OPD into phase difference is shown in Equation (5)

$$
\Delta \varphi_{x}=360 \cdot \Delta L / \lambda
$$

A gradual accumulating phase by supplying a phase difference $\Delta \varphi_{x}$, between adjacent unit cells $\mathrm{X}_{(\mathrm{j})}$ and $\mathrm{X}_{(j+1)}$ in $\hat{\mathrm{x}}$ axis, yields to the desired steering angle $\theta$ in $\mathrm{XZ}$ plane. Using Equations (4) and (5), the connection between $\Delta \varphi_{x}$ and $\Delta x$ to the desired angle steering $\theta$ is shown in Equation (6)

$$
\theta=\sin ^{-1}\left(\lambda \cdot \Delta \varphi_{x} / 360 \cdot \Delta X\right)
$$

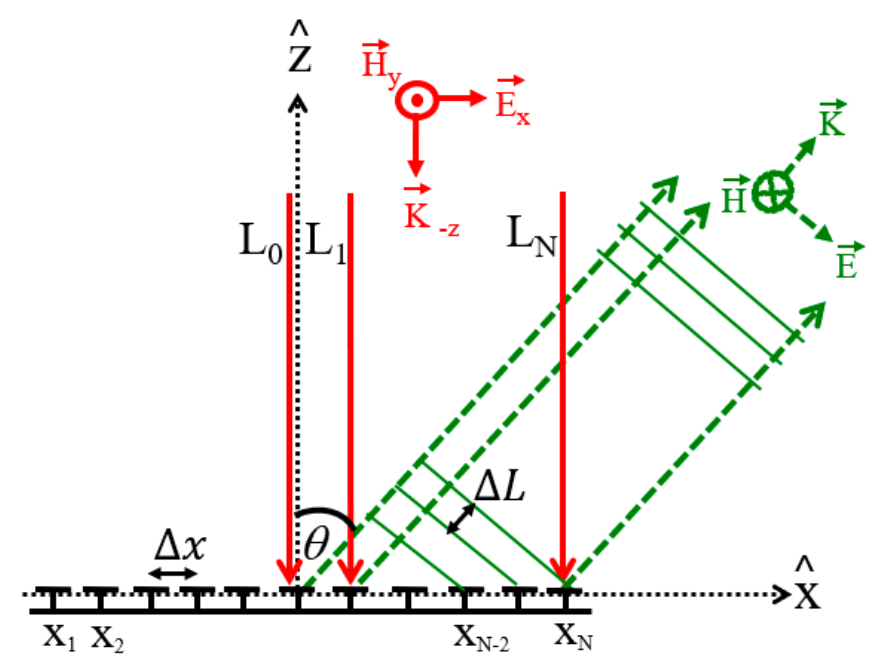

Figure 1. Property of $2-\mathrm{D}$ reconfigurable reflective surface for incident rays $\mathrm{L}_{0}-\mathrm{L}_{\mathrm{N}}$, normal to the metasurface (MS).

For steering angle $\theta$ in the $\mathrm{YZ}$ plane, the same analysis in $\hat{y}$ axis is valid using $\Delta \varphi_{y}$ and $\Delta y$ in Equations (4)-(6). A combination of independent steering in planes $\mathrm{XZ}$ and $\mathrm{YZ}$ results in 2-D steering ability. Those MS properties are frequency-sensitive, allowing us to steer the reflection direction of an incident beam in a limited frequency band.

\section{Unit Cell and MS Reflector Design}

The unit cell design and its equivalent circuit model are shown in Figure 2.

The front-side, backside, profile, and 3-D inside view of two adjacent unit cells are shown in Figure $2 \mathrm{a}-\mathrm{d}$, and the unit cell equivalent circuit model is shown in Figure 2e. The unit cell is composed of two similar dielectric substrates of Rogers Company, model RT/Duroid 5880 with $\varepsilon_{\mathrm{r}}=2.2$ [36], and three copper layers of 35-micrometer thickness. There are two vertical strips in the top layer, each connected to a pad, according to the varactor layout [17]. One of the vertical strips is the same length of the unit cell width-W. The second strip is shorter- $S_{L}-$ and is connected by the via to the circle pad in the lower copper layer, which functions as the DC bias layer. The middle copper layer is for metal ground purposes and separated by clearance from the via which crosses it (see Figure 2d). In a single unit cell, there is a small violation of the $\hat{x}$ axis symmetry, resulting from the use of one via and the different strip lengths. The right unit cell on the $\hat{x}$ axis is a mirror image of the left unit cell, such that symmetric array is obtained (see Figure 2a-d).

$C_{i n t}$ is governed by the radiation electric field component's interaction, with the unit cell strips edges. $C_{\text {int }}$ is inversely proportional to the distance between the strip's edges $D$ [37], as described in Equation (7)

$$
C_{\text {int }}[F / m]=\frac{\pi \varepsilon_{0} \varepsilon_{r}}{\ln \left(4\left(S_{w}+D\right) / S_{w}\right)}
$$


where $S_{W}$ is the strip width. The length $P$ of the unit cell is larger than the width $W$ and allows the strips to be positioned such that $D_{0}$, and $D_{i}$ lead to low intrinsic capacitance (see Figure 2a). According to Equation (7), the calculated capacitances contributions of the strip's edges are $0.0451 \mathrm{pF}$ and $0.0503 \mathrm{pF}$ for $D_{i}=0.9 \mathrm{~mm}$ and $D_{o}=0.6 \mathrm{~mm}$, respectively, and sum up to $C_{i n t}=0.0954 \mathrm{pF}$. Thus, the capacitance and coupling between adjacent unit cells decrease. This geometry enables operation at Ka-band frequencies, with sufficient surface area for the varactor integration, preventing significant absorbing and diffusing. The reduction in $S_{w}$ increases the distance between the strips and decreases $C_{i n t}$ but also increases unit cell losses, and therefore is limited. Furthermore, it distorts the uniformity of the electric field distribution on the unit cell and decreases bandwidth [25].

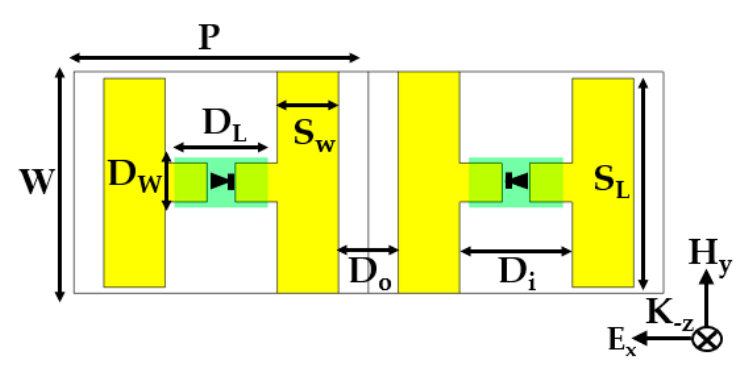

(a)

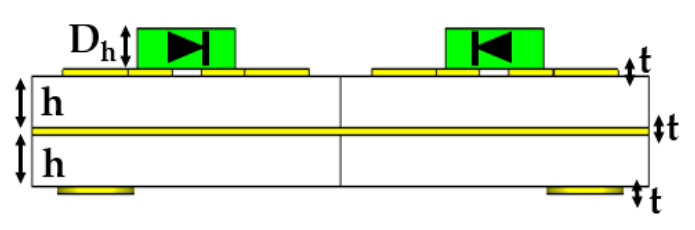

(c)

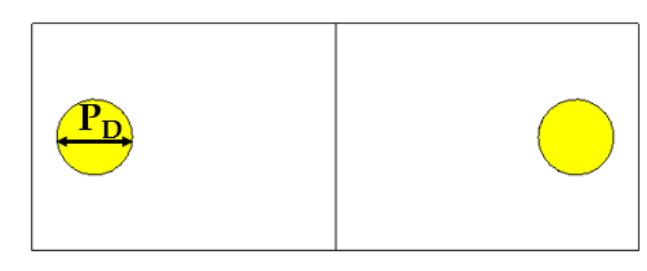

(b)

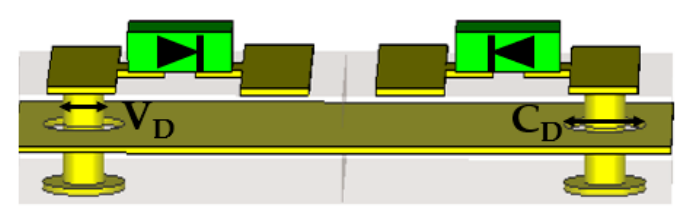

(d)

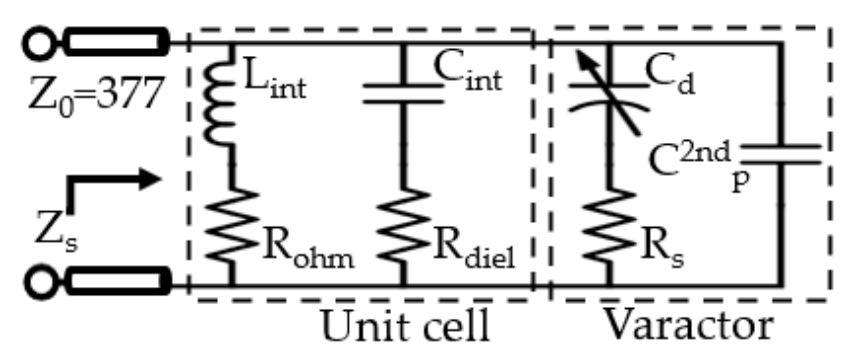

(e)

Figure 2. Two adjacent unit cells design. (a) Unit cell frontside. (b) Unit cell backside. (c) Profile view of the unit cell. (d) Three-dimensional inside view of the unit cell. (e) Unit cell equivalent circuit model.

MACOM varactor diode model MAVR-011020-1411 [17] is used in this MS, which provides extremely low capacitance. The varactor is placed between the strips (see Figure 2 in green), adding variable capacitance $C_{d}$ to the unit cell. The dynamic range of the capacitance $C_{d}$ is $C_{\max }=0.216 \mathrm{pF}$ to $C_{\min }=0.032 \mathrm{pF}$ for $0-15 \mathrm{~V}$ reverse bias voltage, respectively. The package capacitance is included in $C_{d}$ and provides a capacitance ratio of 7 [17]. The MS reflector structure with the DC bias scheme is shown in Figure 3. 


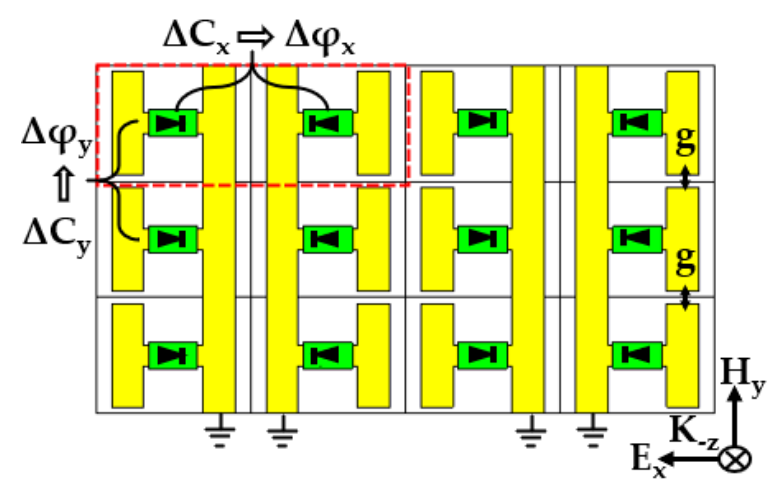

(a)

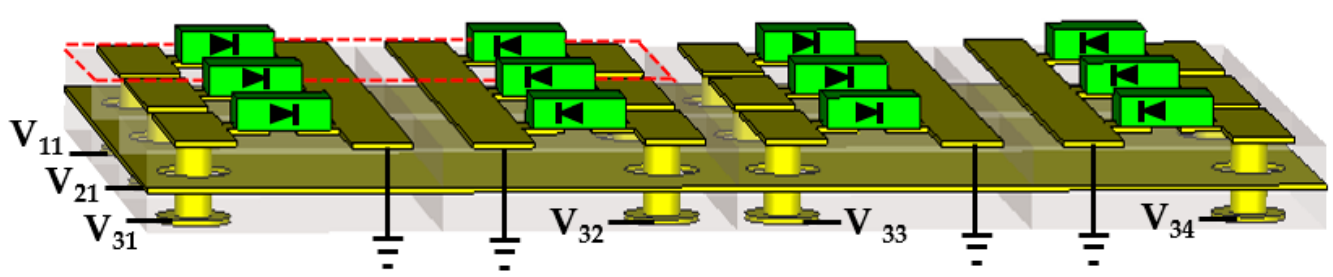

(c)

\begin{tabular}{|l|l|l|l|}
\hline $\mathbf{V}_{11}$ & $\mathbf{v}_{12} \bigcirc$ & $\bigcirc \mathbf{v}_{13}$ & $\mathbf{v}_{14} \bigcirc$ \\
\hline $\mathbf{v}_{21}$ & $\mathbf{v}_{22} \bigcirc$ & $\bigcirc \mathbf{v}_{23}$ & $\mathbf{v}_{24} \bigcirc$ \\
\hline$\bigcirc \mathbf{v}_{31}$ & $\mathbf{v}_{32} \bigcirc$ & $\bigcirc \mathbf{v}_{33}$ & $\mathbf{v}_{34} \bigcirc$ \\
\hline
\end{tabular}

(b)

Figure 3. MS structure with varactors DC bias idea. (a) MS frontside. (b) MS backside with varactors DC bias indexes. (c) Three-dimensional inside view of MS closure of electrical circuit for each varactor.

The MS structure and the simple DC bias method of this geometry, which reduces the complexity and absorption of the unit cell, are shown in Figure 3, where the red dashed rectangles mark the location of a specific two adjacent unit cell. The via and the clearance add losses to the unit cell and are a constraint due to the need to provide DC voltages for each varactor. The longer strip in each unit cell is connected to other unit cell's longer strips throughout the column. These strips function as a common DC ground for the varactors in each column, without the need for an additional via in each unit cell. The shorter strip receives a separate DC voltage from the back of the surface using the via, allowing each MS unit cell to be independently configurable (see Figure 3). The phase reflection is independently manipulated in 2-D, compared to [25], in which the varactors are between the adjacent unit cells, and each unit cell receives only a DC ground or a specific DC voltage. Thus, a constraint is created for the capacitance value between each unit cell and the four unit cells around it. This limitation does not allow the reflection phase to be independently configurable in 2-D. This is an improvement of our previous experimental work, which demonstrated beam steering in 1-D at the working frequencies of 21-23.5 GHz [38]. In this work, a simultaneous and independent 2-D beam steering is presented at a higher working frequency of $37 \mathrm{GHz}$, for the incident polarization, which is shown in Figure 3a.

Considerations in selecting the materials and determining the unit cell's parameters values are described as follows: the selected RT/Duroid 5880 dielectric substrate has a low $\varepsilon_{\mathrm{r}}=2.2$, which supports the need to reduce the $C_{\text {int }}$, according to Equation (7). The substrate thickness is proportional to $L$ [34]. Thus, increasing the substrate thickness increases $B W$ and vice versa, according to Equation (2). The losses are in accordance with the $Q$ factor, which is inversely proportional to $B W$; thus, decreasing the substrate thickness leads to an increase in the acquired dynamic range and higher losses at the working frequency. For the presented unit cell geometry, two commercial dielectric substrate thicknesses of 0.127 and $0.381 \mathrm{~mm}$ were simulated. Low losses of $2.17 \mathrm{~dB}$ were obtained at the working frequency of $37 \mathrm{GHz}$ for a substrate thickness of $0.381 \mathrm{~mm}$, but with low dynamic phase range of only $256^{\circ}$. A high dynamic phase range of $323^{\circ}$ was obtained at the working frequency of $37 \mathrm{GHz}$ for a 
substrate thickness of $0.254 \mathrm{~mm}$, but with a loss of $7.35 \mathrm{~dB}$. Optimization was performed for a substrate thickness of $0.254 \mathrm{~mm}$, to achieve the targets of a loss lower than $5 \mathrm{~dB}$ and a dynamic phase range higher than $300^{\circ}$, at the working frequency of 37. Using 3-D electromagnetic simulation software, CST [39], the unit cell parameters of the simulations were swept with emphasis on $\mathrm{W}$ and $S_{w}$. The final optimized parameters, which are in accordance with the design principles, and suitable to standard PCB manufacturing, are summarized in Table 1.

Table 1. Unit cell parameters.

\begin{tabular}{ccc}
\hline Parameter & Description & Value [mm] \\
\hline $\mathrm{P}$ & Unit cell length & 2.7 \\
$\mathrm{~W}$ & Unit cell width & 1.7 \\
$\mathrm{~S}_{\mathrm{L}}$ & Pad/Line length & 1.573 \\
$\mathrm{~S}_{\mathrm{w}}$ & Pad/Line width & 0.6 \\
$\mathrm{~g}$ & Horizontal gap between the slots & 0.127 \\
$\mathrm{D}_{\mathrm{L}}$ & Varactor diode length & 0.7615 \\
$\mathrm{D}_{\mathrm{w}}$ & Varactor diode width & 0.406 \\
$\mathrm{D}_{\mathrm{H}}$ & Varactor diode height & 0.203 \\
$\mathrm{Di}$ & Distance between outer strips & 0.6 \\
$\mathrm{Do}$ & Distance between inner strips & 0.9 \\
$\mathrm{~h}$ & Dielectric substrate thickness & 0.254 \\
$\mathrm{t}$ & Copper thickness & 0.035 \\
$\mathrm{P}_{\mathrm{D}}$ & Pad diameter & 0.6 \\
$\mathrm{~V}_{\mathrm{D}}$ & Via diameter & 0.3 \\
$\mathrm{C}_{\mathrm{D}}$ & Clearness diameter & 0.6 \\
\hline
\end{tabular}

\section{Simulations Results and Analysis of Unit Cell Reflection and Dynamic Phase Range}

The proposed MS reflector was simulated using the TEM Floquet port with CST simulation software [39]. The reflection simulation of a unit cell as an infinite array for the normal incident corresponds to the polarization described in Figure 2a, as shown in Figure 4.

The simulation results of the unit cell magnitude and phase reflection are shown in Figure 4 as a function of frequency, for the following three capacitance values: $\mathrm{C}_{\mathrm{d} \min }=0.032 \mathrm{pF}, \mathrm{C}_{\mathrm{d} \max }=0.216 \mathrm{pF}$ and $\mathrm{C}_{\mathrm{d}(37 \mathrm{GHz})}=0.065 \mathrm{pF}$, which is the varactor capacitance value that leads to the unit cell resonance frequency at $37 \mathrm{GHz}$. The unit cell resonance frequency is electronically changed from 30.3 to $43.1 \mathrm{GHz}$. It provides a dynamic reflection phase range of up to $303^{\circ}$ and over $300^{\circ}$ in wide bandwidth of over $4 \mathrm{GHz}$, marked between the dashed black crossers at 33.25 and $37.55 \mathrm{GHz}$, as shown in Figure 4b.

The unit cell equivalent circuit model with all the inherent parameters is shown in Figure 2e [7]. The total unit cell resistance $\mathrm{R}$ influences only the absorption losses intensity and is composed of $R_{\text {diel }}$ from dielectric losses, $R_{o h m}$ from ohmic losses, $R_{s}$ from varactor serial resistance, and another resistance component $R_{p}$ from inaccuracies and parasitics in production. While $R_{\text {diel }}$ and $R_{o h m}$ are well defined and quantified in CST simulation, the $R_{s}$ value is unknown, and the $R_{p}$ value depends on the production quality and not on unit cell inherent properties. Based on similar studies $[40,41]$, and an accurate qualitative production assumption, the equivalent value of $R_{s}$ and $R_{p}$ in the simulations was determined as $4 \Omega$.

In this study, careful design and analysis are required due to very small capacities in this unit cell, mainly around $\mathrm{C}_{d}=\mathrm{C}_{\min }$. The varactor physical presence and its contact with the pads (see Figure 2) add parasitic capacitance to the unit cell and should be taken into consideration due to the low $C_{i n t}$ and $C_{d}$ in this realization. We define it as the second-order parasitic capacitance $C^{2 n d}$. This value is influenced by the varactor environment and the varactor effective dielectric constant $\varepsilon_{\text {eff, }}$, which depends on the varactor material. $C^{2 n d}{ }_{p}$ is modeled in CST simulation as a rectangular dielectric slab of the varactor size with estimated value off $\varepsilon_{\text {eff }}$, as shown in green in Figure $2 \mathrm{a}-\mathrm{d}$. The dielectric values of the varactor compounds are silicon nitride-7.65 [42], polyimide-3.44, and gallium arsenide-12.9 (the values from the CST library). The value $\varepsilon_{\text {eff }}=6$, which is around the middle of the extreme value and is set for 
the rectangular dielectric slab. According to the CST simulation, $C^{2 n d}{ }_{p}=0.008 \mathrm{pF}$, a value which is usually negligible but needs to be considered in this work because of the very low total capacitance. The unit cell dynamic capacitance range is shown in Equation (8)

$$
\left(C_{i n t}+C_{d \min }+C^{2 n d} p\right)<C<\left(C_{i n t}+C_{d \max }+C_{p}^{2 n d}\right)
$$

where $C$ is between 0.1354 and $0.3194 \mathrm{pF}$. Plugging $C$ for different cases of $C_{d}$ in (2) shows good agreement in the $f_{\text {res }}$ tuning simulations results, as shown in Figure 4 . The reduction in $C_{i n t}$, combined with the chosen varactor, leads to a high capacitance ratio and allows wide tunability.

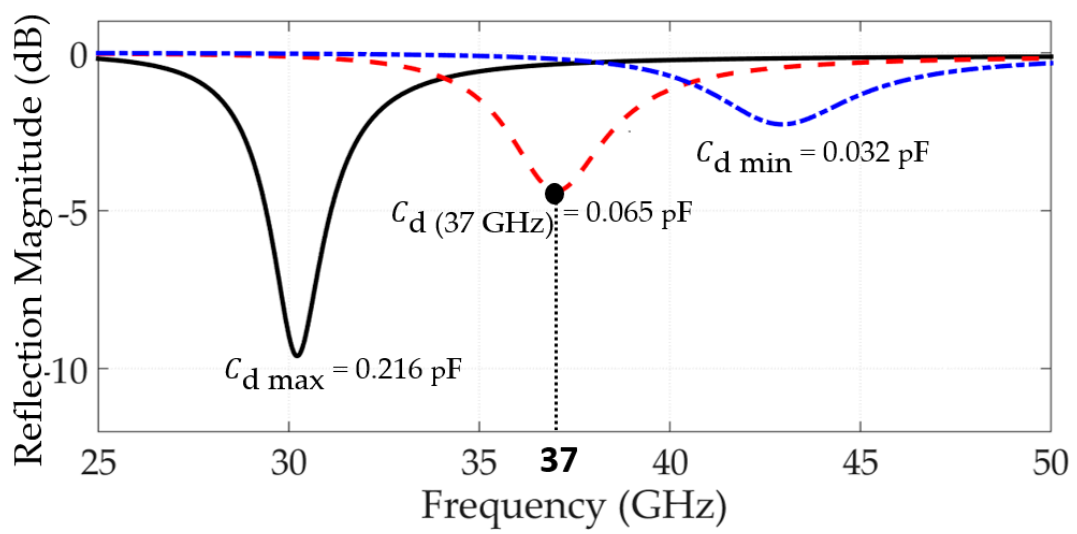

(a)

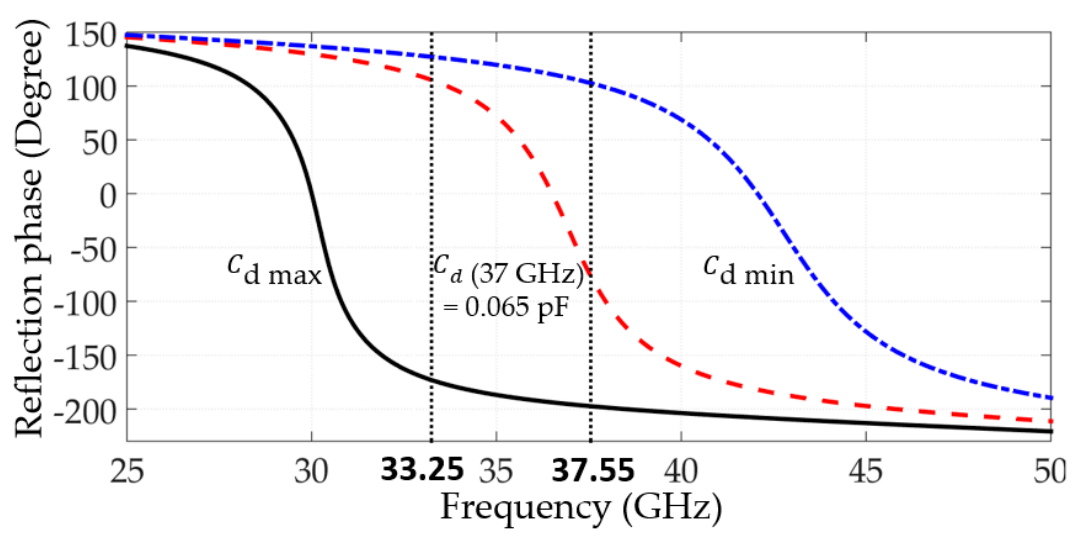

(b)

Figure 4. Simulation results of unit cell reflection. (a) Magnitude. (b) Phase.

Gradual linear accumulated reflected phases throughout the MS reflector with uniform reflected intensities are required to achieve the desired steering angle $\theta$ described in (6). In practice, impurity in the intensity causes a minor change in $\theta$, due to different losses in the MS reflector unit cells. The continuous tuning ability of each unit cell allows using error correction algorithms for improved results and accuracy. The maximum loss value is $4.37 \mathrm{~dB}$ for unit cells in the MS with a resonance frequency of $37 \mathrm{GHz}$, as shown in Figure 4a (middle red line). Losses of unit cells with higher or lower resonance frequency are less than $4.37 \mathrm{~dB}$ at $37 \mathrm{GHz}$ with a negligible value for unit cells with resonance frequencies, which relate to $C_{d \max }$ and $C_{d \text { min }}$. This phenomenon of losses is unavoidable due to the nature of resonance element usage, but is minimized by using low loss materials and proper unit cell design, such as decreasing $Q$ factor.

Sweeping the above unit cell reflection phase simulations versus varactor capacitance $C_{d}$ at specific work frequency $37 \mathrm{GHz}$ leads to the dynamic phase range as shown in Figure 5. 


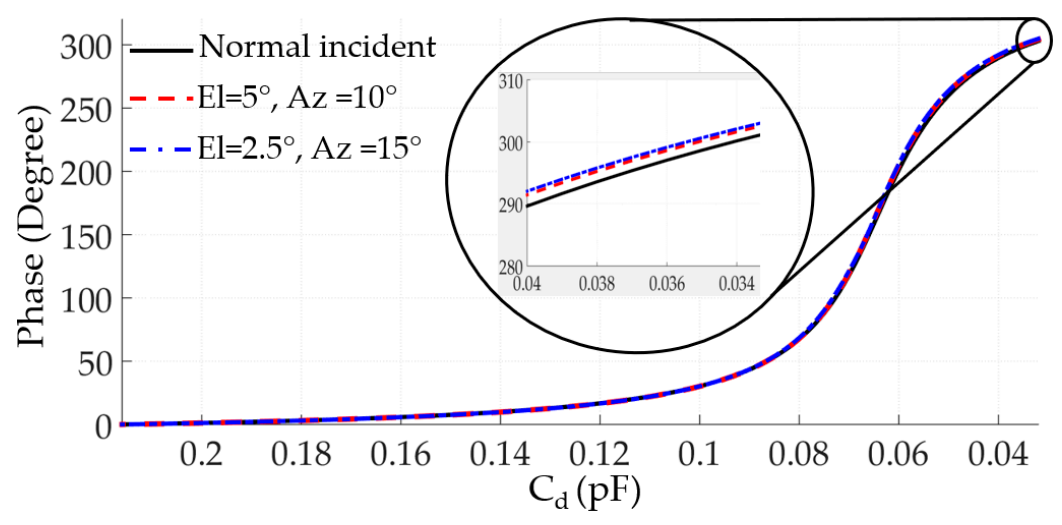

Figure 5. Unit cell dynamic range of reflection phase as a function of the $C_{d}$ at $37 \mathrm{GHz}$ for normal incident and two extreme off-normal incident cases.

Figure 5 shows the whole dynamic phase range of unit cell reflection phase at $37 \mathrm{GHz}$ as a function of $C_{d}$, for a normal incident (in black line) and off-normal incidents (in red dashed and blue dotted-dashed lines). The DC bias for each unit cell is determined based on the calibration curve in Figure 5, such that the $\Delta C_{x}$ between adjacent unit cells in the $\hat{x}$ axis provides the desired $\Delta \varphi_{\mathrm{x}}$, and the $\Delta C_{y}$ between adjacent unit cells in the $\hat{y}$ axis provides the desired $\Delta \varphi_{y}$, as seen in Figure $3 a$. Phase values were normalized between $0^{\circ}$ and $360^{\circ}$ with a value of $0^{\circ}$ for $C_{d \text { max }}$, and $303^{\circ}$ for $C_{d \text { min }}$ and allow MS spatial phase distribution value of $303^{\circ}$ out of the ideal value of $360^{\circ}$, where the dynamic phase is used in a cyclic manner. For high steering $\theta$, which requires $\Delta \varphi \geq 57^{\circ}$ according to (6) or for large size MS, which requires the usage of more than $303^{\circ}$ available range, the range part of $57^{\circ}$ leads to a phase error. The fundamental array constant $\Delta \mathrm{x}=P$ or $\Delta \mathrm{y}=W$ can be doubled to reduce the influence of the missing phase range on $\Delta \varphi$ in (6). This doubling increases the effective array constant parameters and compensates over the limitation in $\Delta \varphi$ values. The multiplication of array constants $\Delta \mathrm{x}$ and $\Delta \mathrm{y}$ is achieved by applying the same DC voltage/capacitance value to adjacent unit cells in the $\hat{x}$ or the $\hat{y}$ axis, respectively, as seen in Figure 3a,b. The array constants $\Delta x$ and $\Delta y$ can also be multiplied further where a higher value leads to exceeding the MS definition, where unit cell dimensions are smaller than the working wavelength. Alternatively, any steering can be achieved without multiplying $\Delta x$ or $\Delta y$ with performance degradation due to phase mismatch [43]. For small-sized MS, $\Delta \varphi$ can be used without limitation, as long as it is used in one dynamic phase range cycle. This is a typical limitation of MS reflector, which is seen in other lower frequency studies of different geometries $[25,29,30]$.

\section{Reconfigurable MS Reflector Steering Results}

The final MS reflector is a fixed 2-D array size that was designed and simulated based on the above unit cell reflection results. The array size is $75.2 \mathrm{~mm} \times 188 \mathrm{~mm}$ with 12 rows and 8 columns of unit cells, which sum up to 96-unit cells, and can be stimulated with separate DC voltages as needed. MS reflector with the axes steering definition and an example of a 3-D far-field reflection result, without phase manipulation, are shown in Figure 6.

For azimuth steering $(-\hat{x})$, every unit cell in each column is connected to the same DC voltage/capacitance value, to achieve the desired $\Delta \varphi_{x}$ while $\Delta \varphi_{y}=0$, and for elevation steering $(\hat{y})$, every unit cell in each row gets the same DC voltage/capacitance value, to achieve the desired $\Delta \varphi_{y}$ while $\Delta \varphi_{x}=0$.

For 1-D steering mode, considering the dynamic phase range and MS size, the phase difference parameter- $\Delta \varphi_{x}$ or $\Delta \varphi_{y}$-is limited, as shown in Equation (9)

$$
303^{\circ} \geq 7 \times \Delta \varphi_{x}, 11 \times \Delta \varphi_{y}
$$


For azimuth and elevation 1-D steering, several $\theta$ angles were calculated using (6) and simulated using CST for normal incident TEM plan wave around $37 \mathrm{GHz}$, which corresponds to the polarization described in Figure 6. The simulation results are shown in Figure 7.

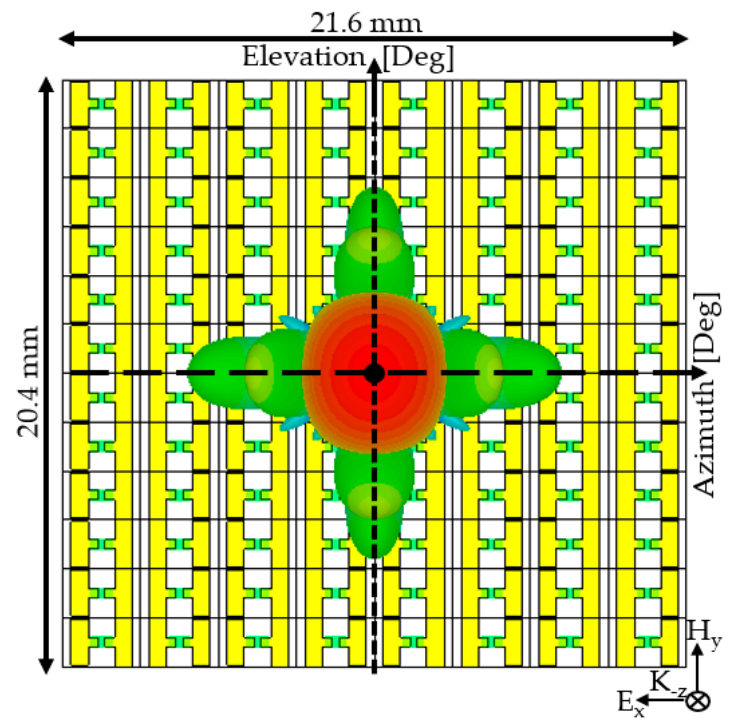

Figure 6. Final array dimensions, with a 3-D far-field result of the reference case.

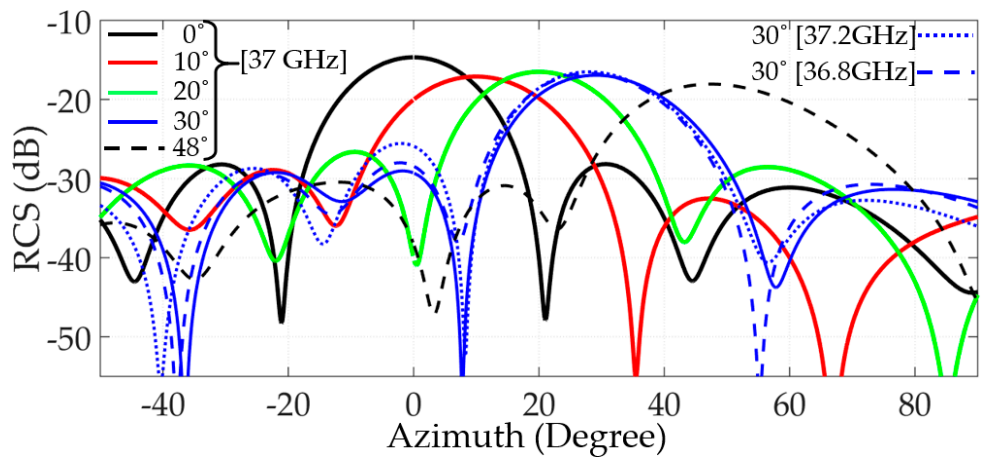

(a)

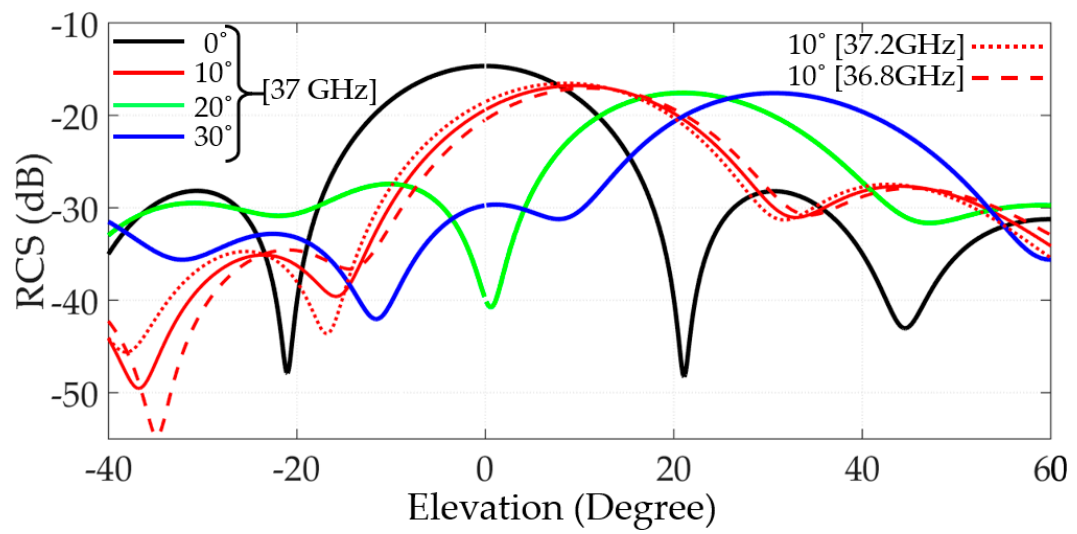

(b)

Figure 7. Results of far-field Radar Cross Section (RCS) for beam steering at $37 \mathrm{GHz}$ in solid lines, where the black line is without phase manipulation for comparison. Steering results around $37 \mathrm{GHz}$ in discontinuous lines. (a) Beam steering in azimuth axis. (b) Beam steering in elevation axis. 
Steering angles simulations results of $10^{\circ}, 20^{\circ}, 30^{\circ}$, and $48^{\circ}$ in azimuth and $10^{\circ}, 20^{\circ}$, and $30^{\circ}$ in elevation compared to reference result at $0^{\circ}$ are shown in Figure $7 \mathrm{a}, \mathrm{b}$, respectively.

Deviation for $\pm 200 \mathrm{MHz}$ around $37 \mathrm{GHz}$ for the same TEM incident wave for steering angle $30^{\circ}$ in azimuth and $10^{\circ}$ in elevation is shown in Figure 7 (dotted and dashed lines). The results show overlap within frequency deviations, which leads to a bandwidth of about $400 \mathrm{MHz}$.

Tables 2 and 3 summarize the parameters in Equation (6) for calculating the steering angles shown in Figure 7.

Table 2. Value parameters in Equation (6) for Azimuth steering angles $\theta$ calculations.

\begin{tabular}{ccc}
\hline Azimuth $[\mathrm{Deg}]$ & $\boldsymbol{\Delta} \boldsymbol{\varphi}_{\mathbf{x}}[\mathrm{Deg}]$ & $\boldsymbol{\Delta \mathbf { x }}[\mathbf{m m}]$ \\
\hline 10 & 20.83 & 2.7 \\
20 & 41.04 & 2.7 \\
30 & 60 & 2.7 \\
48 & 90 & 2.7 \\
\hline
\end{tabular}

Table 3. Value parameters in (6) for Elevation steering angles $\theta$ calculations.

\begin{tabular}{ccc}
\hline Elevation $[\mathrm{Deg}]$ & $\Delta \boldsymbol{\varphi}_{\mathbf{y}}[\mathrm{Deg}]$ & $\Delta \mathrm{y}[\mathrm{mm}]$ \\
\hline 10 & 13.12 & 1.7 \\
20 & 25.84 & 1.7 \\
30 & 75.55 & 3.4 \\
\hline
\end{tabular}

In 2-D steering mode, considering the dynamic phase range and MS size, the phase difference parameters, $\Delta \varphi_{x}$ and $\Delta \varphi_{y}$, are limited, as shown in Equation (10)

$$
303^{\circ} \geq 7 \times \Delta \varphi_{x}+11 \times \Delta \varphi_{y}
$$

In order to serve an angular spatial cone of $20^{\circ}$ and $10^{\circ}$, steering abilities of $\pm 10^{\circ}$ in azimuth and $\pm 5^{\circ}$ in elevation are required and lead to maximum values of $\Delta \varphi_{\mathrm{x}}=20.83^{\circ}$ and $\Delta \varphi_{\mathrm{y}}=6^{\circ}$, respectively. The total sum of MS phase distribution in use is $211.81^{\circ}$, which is smaller than $303^{\circ}$ and meets the definition in Equation (10). In order to serve an angular spatial cone of $30^{\circ}$ and $5^{\circ}$, steering abilities of $\pm 15^{\circ}$ in azimuth and $\pm 2.5^{\circ}$ in elevation are required and lead to maximum values of $\Delta \varphi_{\mathrm{x}}=31.05^{\circ}$ and $\Delta \varphi_{\mathrm{y}}=3.3^{\circ}$, respectively. The total sum of MS phase distribution in use is $253.65^{\circ}$, which also meets the definition in (10).

Figure 7 shows the simulation results of the dynamic phase range for off-normal incident radiation, which comes from the above spatial cone example's edges compared to the simulation results for a normal incident case. The simulation results coincide. Thus, a reasonable spatial cone can be supported and covered by the MS reflector. Two-dimensional steering simulation results at $37 \mathrm{GHz}$ for the angular partial cone edges mentioned cases are shown in Figure 8.

Following the discussion in paragraph 5, the use of Equation (6) in the case of non-uniformed intensities, due to different losses in MS reflector unit cells, leads to a small gap between the simulated steering angles $\theta$, shown in Figure 8, compared to the calculated steering angles $\theta$, summarized in Table 4 . This gap can be corrected by a slight change in the phase difference parameters $-\Delta \varphi_{x}$ and $\Delta \varphi_{y}$-used in Equation (6). Due to MS reflector symmetry, the same steering results in 1-D and 2-D can be obtained in the opposite direction, by inversing the capacitance and phase distribution gradient. Comparison between continuous tunable MS reflector works with a varactor as a tuning method is presented in Table 5. 


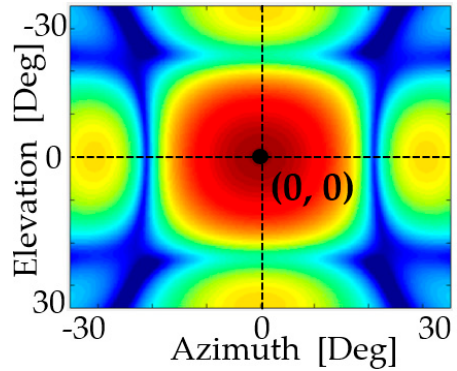

(a)

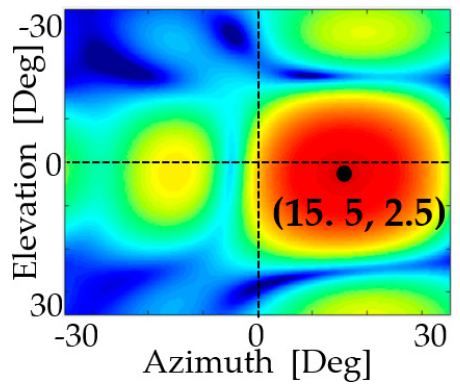

(d)

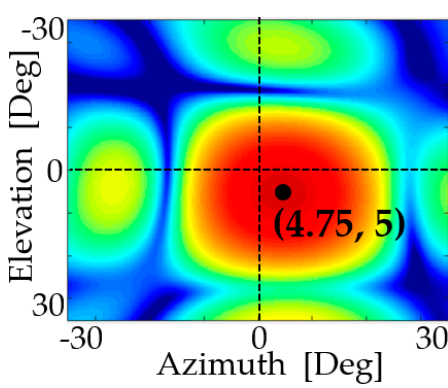

(b)

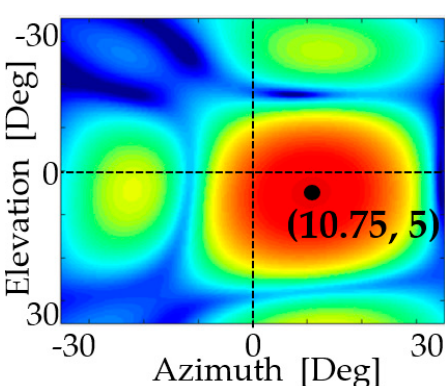

(c)

Figure 8. Two-dimensional far-field beam steering results for several steering in azimuth and elevation simultaneously. (a) Reference result. (b-e) Two-dimensional steering results.

Table 4. Value parameters in (6) for 2-D steering angles $\theta$ calculations.

\begin{tabular}{ccc}
\hline Az, El [Deg] & $\Delta \varphi_{\mathbf{x}}, \Delta \varphi_{\mathbf{y}}[\mathrm{Deg}]$ & $\Delta \mathbf{x}, \Delta \mathbf{y}[\mathrm{mm}]$ \\
\hline 5,5 & 10,6 & $2.7,1.7$ \\
10,5 & $20.83,6$ & $2.7,1.7$ \\
$7.5,2.5$ & $15.66,3.3$ & $2.7,1.7$ \\
$15,2.5$ & $31.05,3.3$ & $2.7,1.7$ \\
\hline
\end{tabular}

Table 5. Abilities comparison between MS reflector works.

\begin{tabular}{ccccccc}
\hline Reference & $\begin{array}{c}\text { Frequency } \\
\text { (GHz) }\end{array}$ & $\begin{array}{c}\text { Phase } \\
\text { Range (Deg) }\end{array}$ & $\begin{array}{c}\text { 1-D Steering Range } \\
\text { (Deg) }\end{array}$ & $\begin{array}{c}\text { 2-D Steering Range } \\
\text { (Deg) }\end{array}$ & 1 $\backslash$ 2 Polarization & Measurement $\backslash$ Simulation \\
\hline$[25]$ & 4.5 & $325^{*}$ & 40 & Not presented & 2 separately & Meas \\
{$[28]$} & $9-11$ & 290 & 45 & Not presented & 1 & Meas \\
{$[29]$} & 12.1 & $300^{*}$ & 56 & Not presented & 1 & Sim \\
{$[30]$} & 15 & 330 & 63 & Not presented & 1 & Sim \\
{$[38]$} & $21-23.5$ & 320 & 57 & $10 / 5$ & 1 & Meas \\
This work & 37 & 303 & 48 & Sim & \\
\hline
\end{tabular}

* Estimated value from a graph [25].

\section{Conclusions}

The concept and simulations of the MS reflector for $5 \mathrm{G}$ wireless communication based on a new resonant unit cell are presented. This unit cell offers a phase dynamic range of up to $303^{\circ}$ and above $300^{\circ}$ in Ka-band in more than $4 \mathrm{GHz}$ bandwidth with relatively low losses, which allow 2-D beam steering (azimuth and elevation) with about $400 \mathrm{MHz}$ bandwidth simulated at $37 \mathrm{GHz}$. The unit cell design fits the standard PCB technology using off-the-shelf products. Engineering limitations and parameters arising from phase sensitivity and high frequency were analyzed, such as resistance component from inaccuracies and parasitics in production and second-order parasitic capacitance phenomena from varactor assembly. There is a trade-off between the losses and the phase dynamic range of the unit cell. Optimization was performed on the unit cell parameters for a target of losses lower than $5 \mathrm{~dB}$ and a phase dynamic range higher than $300^{\circ}$ at $37 \mathrm{GHz}$, one of the emerging $5 \mathrm{G}$ 
frequencies. Thus, a large dynamic phase range was achieved while maintaining relatively low losses, which improve reflector performance.

Author Contributions: Conceptualization, D.R.; methodology, D.R. and A.A.; software, D.R. and E.R.; validation, D.R.; data curation, D.R.; formal analysis, D.R.; investigation, D.R. and E.R.; resources, D.R and A.A.; writing - original draft preparation, D.R.; writing—review and editing, D.R., E.R. and A.A.; visualization, D.R.; supervision, A.A.; project administration, D.R.; funding acquisition, A.A. All authors have read and agreed to the published version of the manuscript.

Funding: This research received no external funding.

Conflicts of Interest: The authors declare no conflict of interest.

\section{References}

1. Rappaport, T.S.; Xing, Y.; MacCartney, G.R.; Molisch, A.F.; Mellios, E.; Zhang, J. Overview of Millimeter Wave Communications for Fifth-Generation (5G) Wireless Networks-With a Focus on Propagation Models. IEEE Trans. Antennas Propag. 2017, 65, 6213-6230. [CrossRef]

2. Hong, T.; Yao, J.; Liu, C.; Qi, F. mmWave Measurement of RF Reflectors for 5G Green Communications. Wirel. Commun. Mob. Comput. 2018, 2018, 8217839. [CrossRef]

3. Peng, Z.; Li, L.; Wang, M.; Zhang, Z.; Liu, Q.; Liu, Y.; Liu, R. An effective coverage scheme with passive-reflectors for urban millimeter-wave communication. IEEE Antennas Wirel. Propag. 2015, 15, 398-401. [CrossRef]

4. Zhang, L.; Chen, X.; Fang, Y.; Huang, X.; Fang, X. Learning-Based mmWave V2I Environment Augmentation through Tunable Reflectors. In Proceedings of the 2019 IEEE Global Communications Conference (GLOBECOM), Waikoloa, HI, USA, 9-13 December 2019.

5. Khawaja, W.; Ozdemir, O.; Yapici, Y.; Guvenc, I.; Kakishima, Y. Coverage Enhancement for mmWave Communications Using Passive Reflectors. In Proceedings of the 2018 11th Global Symposium on Millimeter Waves (GSMM), Boulder, CO, USA, 22-24 May 2018.

6. Giordani, M.; Zorzi, M. Improved User Tracking in 5G Millimeter Wave Mobile Networks via Refinement Operations. In Proceedings of the 2017 16th Annual Mediterranean Ad Hoc Networking Workshop (Med-Hoc-Net), Budva, Montenegro, 28-30 June 2017.

7. Li, A.; Singh, S.; Sievenpiper, D. Metasurfaces and their applications. Nanophotonics 2018, 7, 989-1011. [CrossRef]

8. Rotshild, D.; Azoulay, D.Y.; Ochana, M.; Shulzinger, A.; Abramovich, A. Real time detection and recognition of micro-poisons in aqueous solutions and atmosphere using perfect absorber metamaterial in millimeter wavelength regime. In Proceedings of the 2015 IEEE International Conference on Microwaves, Communications, Antennas and Electronic Systems (COMCAS), Tel Aviv, Israel, 2-4 November 2015.

9. Litmanovitch, G.; Rotshild, D.; Abramovich, A. Flat mirror for millimeter-wave and terahertz imaging systems using an inexpensive metasurface. Chin. Opt. Lett. 2017, 15, 011101. [CrossRef]

10. Hum, S.V.; Perruisseau-Carrier, J. Reconfigurable reflectarrays and array lenses for dynamic antenna beam control. IEEE Trans. Antennas Propag. 2013, 62, 183-198. [CrossRef]

11. Mavridou, M.; Feresidis, A.P. Dynamically reconfigurable high impedance and frequency selective metasurfaces using piezoelectric actuators. IEEE Trans. Antennas Propag. 2016, 64, 5190-5197. [CrossRef]

12. Kamoda, H.; Iwasaki, T.; Tsumochi, J.; Kuki, T. 60-GHz electrically reconfigurable reflectarray using p-i-n diode. In Proceedings of the 2009 IEEE MTT-S International Microwave Symposium Digest, Boston, MA, USA, 7-12 June 2009.

13. Solderable AlGaAs Flip Chip PIN. Available online: https://cdn.macom.com/datasheets/MADP-00090714020x.pdf (accessed on 11 June 2020).

14. Tian, W.; Li, P.; Yuan, L. Research and analysis of MEMS switches in different frequency bands. Micromachines 2018, 9, 185. [CrossRef]

15. Foo, S. Liquid-crystal-tunable metasurface antennas. In Proceedings of the 2017 11th European Conference on Antennas and Propagation (EUCAP), Paris, France, 19-24 March 2017.

16. Yaghmaee, P.; Karabey, O.H.; Bates, B.; Fumeaux, C.; Jakoby, R. Electrically tuned microwave devices using liquid crystal technology. Int. J. Antennas Propag. 2013, 2013, 824214. [CrossRef] 
17. MACOM. MAVR-011020-1411, Solderable GaAs Constant Gamma Flip-Chip Varactor Diode. Available online: https://cdn.macom.com/datasheets/MAVR-011020-1141.pdf (accessed on 11 June 2020).

18. Yang, H.; Yang, F.; Cao, X.; Xu, S.; Gao, J.; Chen, X.; Li, M.; Li, T. A 1600-element dual-frequency electronically reconfigurable reflectarray at X/Ku-band. IEEE Trans. Antennas Propag. 2017, 65, 3024-3032. [CrossRef]

19. Guclu, C.; Perruisseau-Carrier, J.; Civi, O. Proof of concept of a dualband circularly-polarized RF MEMS beam-switching reflectarray. IEEE Trans. Antennas Propag. 2012, 60, 5451-5455. [CrossRef]

20. Chaimool, S.; Hongnara, T.; Rakluea, C.; Akkaraekthalin, P.; Zhao, Y. Design of a PIN diode-based reconfigurable metasurface antenna for beam switching applications. Int. J. Antenn. Propag. 2019, 2019, 7216324. [CrossRef]

21. Jang, T.; Lim, S. Novel capacitor-loaded substrate-integrated-waveguide structure and its electronically controlled leaky-wave antenna application. Electromagnetics 2014, 34, 585-592. [CrossRef]

22. Yang, Z.; Zhu, B.O.; Feng, Y. Free Space Electromagnetic Wave Modulation Using Metasurface Absorber. In Proceedings of the 2015 Asia-Pacific Microwave Conference (APMC), Nanjing, China, 6-9 December 2015.

23. Wang, Z.; Liao, D.; Zhang, T.; Chen, T.; Ruan, Y.; Zheng, B. Metasurface-based focus-tunable mirror. Opt. Express 2019, 27, 30332-30339. [CrossRef] [PubMed]

24. Wu, Z.; Ra'di, Y.; Grbic, A. Tunable metasurfaces: A polarization rotator design. Phys. Rev. X 2019, 9, 011036. [CrossRef]

25. Sievenpiper, D.; Schaffner, J.; Song, H.J.; Loo, R.; Tangonan, G. Two-dimensional beam steering using an electrically tunable impedance surface. IEEE Trans. Antennas Propag. 2003, 51, 2713-2722. [CrossRef]

26. Liang, B.; Sanz-Izquierdo, B.; Parker, E.A.; Batchelor, J.C. A Frequency and Polarization Reconfigurable Circularly Polarized Antenna Using Active EBG Structure for Satellite Navigation. IEEE Trans. Antennas Propag. 2015, 63, 33-40. [CrossRef]

27. Kitayama, D.; Yaita, M.; Song, H.-J.; Nosaka, H. High-speed and high-ON/OFF ratio split-ring-resonator-based active metamaterial using varactor diodes. In Proceedings of the 2016 41st International Conference on Infrared, Millimeter, and Terahertz waves (IRMMW-THz), Copenhagen, Denmark, 25-30 September 2016.

28. Ratni, B.; de Lustrac, A.; Piau, G.P.; Burokur, S.N. Active metasurface for reconfigurable reflectors. Appl. Phys. A 2018, 124, 104. [CrossRef]

29. Nie, J.; Tan, Y.Q.; Ji, C.; Liu, R.P. Analysis of Ku-Band Steerable Metamaterials Reflectarray with Tunable Varactors. In Proceedings of the 2016 Progress in Electromagnetic Research Symposium (PIERS), Shanghai, China, 8-11 August 2016; pp. 709-713.

30. Rotshild, D.; Abramovich, A. Wideband reconfigurable entire Ku-band metasurface beam-steerable reflector for satellite communications. IET Microw. Antennas Propag. 2018, 13, 334-339. [CrossRef]

31. Pozar, D.M. Microwave Engineering, 4th ed.; John Wiley \& Sons, Inc.: Hoboken, NJ, USA, 2012; pp. $272-277$.

32. Rea, S.P.; Linton, D.; Orr, E.; McConnell, J. Broadband high-impedance surface design for aircraft HIRF protection. IEE Proc. Microw. Antennas Propag. 2006, 153, 307-313. [CrossRef]

33. Kuse, R.; Hori, T.; Fujimoto, M. PMC and EBG characteristics of cross type artificial magnetic conductor. In Proceedings of the 2012 International Symposium on Antennas and Propagation (ISAP), Nagoys, Japan, 29 October-2 November 2012.

34. Sievenpiper, D. High-Impedance Electromagnetic Surfaces. Ph.D. Thesis, University of California, Los Angeles, CA, USA, 1999; pp. 31-39, 42-44, 47-49.

35. Bhattacharyya, A.K. Phased Array Antennas: Floquet Analysis, Synthesis, BFNs and Active Array Systems; John Wiley \& Sons Press: Hoboken, NJ, USA, 2006; pp. 18-21.

36. Rogers Corporation. RT/Duroid@5880LZ. Available online: https://www.rogerscorp.com/-/media/ project/rogerscorp/documents/advanced-connectivity-solutions/english/data-sheets/rt-duroid-5880lzhigh-frequency-laminates.pdf (accessed on 11 June 2020).

37. Mutual Inductance and Capacitance Algorithm. Available online: https://cecas.clemson.edu/cvel/emc/ expert_systems/PCB/summaries/Mutual_LC_summary.pdf (accessed on 11 June 2020).

38. Rotshild, D.; Abramovich, A. Realization and validation of continuous tunable metasurface for high resolution beam steering reflector at K-band frequency. Int. J. RF Microw. Comput.-Aided Eng.. Under Review.

39. Rütschlin, M.; Wittig, T.; Iluz, Z. Phased antenna array design with CST STUDIO SUITE. In Proceedings of the 2016 10th European Conference on Antennas Propag (EuCAP), Davos, Switzerland, 10-15 April 2016; pp. 1-5. 
40. Wang, M.; Ma, F.W.; Tang, W.X.; Zhang, H.C.; Wang, X.Z.; Cui, T.J. Programmable controls of multiple modes of spoof surface plasmon polaritons to reach reconfigurable plasmonic devices. Adv. Mater. Technol. 2019, 4, 1800603. [CrossRef]

41. Wang, M.; Ma, F.W.; Tang, W.X.; Zhang, H.C.; Wang, X.Z.; Cui, T.H.; Jiang, W.X. A dual-band electronic-scanning leaky-wave antenna based on a corrugated microstrip line. IEEE Trans. Antennas Propag. 2019, 67, 3433-3438. [CrossRef]

42. Zhang, T.; Zhang, S.R.; Wu, M.Q.; Sang, W.J.; Gao, Z.P.; Li, Z.P. Studies on dielectric properties of silicon nitride at high temperature. J. Electron. Sci. Technol. 2007, 5, 316-319.

43. Boriskin, A.; Sauleau, R. (Eds.) Aperture Antennas for Millimeter and Sub-Millimeter Wave Applications; Springer: New York, NY, USA, 2017; pp. 146-150.

(C) 2020 by the authors. Licensee MDPI, Basel, Switzerland. This article is an open access article distributed under the terms and conditions of the Creative Commons Attribution (CC BY) license (http://creativecommons.org/licenses/by/4.0/). 